\title{
DOUBLING METRIC SPACES ARE CHARACTERIZED BY A LEMMA OF BENJAMINI AND SCHRAMM
}

\author{
JAMES T. GILL
}

(Communicated by Jeremy Tyson)

Abstract. A useful property of $\mathbb{R}^{n}$ originally shown by I. Benjamini and O.

Schramm turns out to characterize doubling metric spaces

\section{INTRODUCTION}

In 3], I. Benjamini and O. Schramm prove that the distributional limit of rooted random unbiased finite planar graphs with uniformly bounded degree is almost surely recurrent. By rooted they meant that each graph had a distinguished vertex, and by unbiased they meant that (essentially) given an unrooted graph each vertex is equally likely to be the root. The distributional limit is the weak limit of the random graphs with respect to the topology of local graph isomorphisms in neighborhoods of the root. In this influential paper, they prove a curious lemma which we repeat as Lemma A below.

We use the common notation that in metric space $(X, d)$, the ball $B(x, r)$ consists of all points $y \in X$ such that $d(x, y)<r$. When $K>0$ is positive, we use $K B$ to denote a ball of the same center as $B$, but with radius $K$ times the original.

Definition 1. Let $C$ be a finite set of points of the metric space $(X, d)$. The isolation radius of $w \in C$ is

$$
\rho_{w}=\min \{d(w, v): v \in C \backslash\{w\}\} .
$$

The point $w$ is said to be $(\delta, s)$-supported for some $\delta \in(0,1)$ and $s \geq 2$ if

$$
\inf _{p \in X}\left|\left(B\left(w, \rho_{w} / \delta\right) \backslash B\left(p, \delta \rho_{w}\right)\right) \cap C\right| \geq s .
$$

Lemma A (Benjamini and Schramm, 3. Lemma 2.3). For every $\delta \in(0,1)$ there is a constant $c=c(\delta)$ such that for every finite $C \subset \mathbb{R}^{2}$ and every $s \geq 2$ the set of $(\delta, s)$-supported points in $C$ has cardinality at most $c|C| / s$.

Their proof is valid for any $\mathbb{R}^{n}$ and uses both probability and scaling properties of $\mathbb{R}^{n}$. This lemma is a key step in the proof of their recurrence result mentioned above. It is also known that for $\mathbb{R}^{n}$ that $c(\delta)$ is of the order $\delta^{-n} \log (1 / \delta)$ (see Lemma 3.4 of [5]) . The current author and S. Rohde used this lemma in the proof that Brownian motion on the uniform infinite planar triangulation is recurrent [6].

Received by the editors November 14, 2012 and, in revised form, January 23, 2013 and January $28,2013$.

2010 Mathematics Subject Classification. Primary 30L05; Secondary 28A75.

Key words and phrases. Doubling space, Assouad dimension.

The author was supported by an NSF Mathematical Sciences Postdoctoral Research Fellowship DMS-1004721. 
In 2], I. Benjamini and N. Curien use this same lemma as part of the proof of a result similar to that in 3 but in higher dimensions and more related to sphere packing than to random walks. In [10], H. Namazi, P. Pankka, and J. Souto found the lemma useful to show a certain non-degeneracy of the distributional limit of random Riemannian manifolds with a curvature bound. Finally, in 5, O. GurelGurevich and A. Nachmias proved that the random walk on uniform infinite planar triangulation is recurrent with this now almost "magical" lemma playing a key role.

The usefulness of Lemma A together with its strange opacity leads one to wonder in what settings besides $\mathbb{R}^{n}$ it might hold. This thought naturally produces the following definition.

Definition 2. A metric space $(X, d)$ is said to carry a Benjamini-Schramm lemma if for all $\delta \in(0,1)$ there exists a constant $c=c(X, \delta)$ so that for every finite set $C$ in $X$ and every $s \geq 2$, the number of $(\delta, s)$-supported points in $C$ is less than $c|C| / s$ (i.e., the proportion of points that are $(\delta, s)$-supported is $c / s$ where the constant depends only on delta and, of course, the underlying metric space).

One might also think to allow the decay to depend on other functions of $s$, but the proof of Proposition 3 below shows this is unnecessary. As Lemma A fails spectacularly in an infinite dimensional normed linear space, some concept of finite dimensionality of our space is needed. The following definition is a form of finite dimensionality for metric spaces.

Definition 3. A metric space $(X, d)$ is called doubling if there is a constant $D \geq 1$ so that every set of diameter $d$ in $X$ can be covered by at most $D$ sets of diameter at most $d / 2$.

We note that there are many equivalent definitions of doubling (metric) spaces. In particular, in this note we will force the arbitrary covering sets to be metric balls. One may also choose any number $l>1$ and demand that the covering balls be of diameter at most $d / l$. Euclidean spaces are clearly doubling. Hyperbolic spaces, however, are not. A non-trivial example of a doubling metric space is the Heisenberg group endowed with the Carnot metric. It is metric doubling, but not bi-Lipschitz equivalent to $\mathbb{R}^{n}$. These particulars are discussed in the book [7] (esp. Chapter 10), and more extensively in the paper [ 8 . A metric space that is doubling is often called a space of finite Assouad dimension, which is defined and discussed in Section 1.4.4 of [9]. It turns out that this is precisely the assumption needed for a Benjamini-Schramm lemma.

Theorem 1. A metric space $(X, d)$ carries a Benjamini-Schramm lemma if and only if $(X, d)$ is doubling.

\section{Proof of Equivalence}

We prove Theorem 1 through a series of propositions. The following proposition is essentially part of the proof of Lemma 5.1 in [10].

Proposition 1. If $(X, d)$ carries a bi-Lipschitz embedding into $\mathbb{R}^{n}$ then $(X, d)$ carries a Benjamini-Schramm lemma.

Proof. Let us denote the bi-Lipschitz embedding by $f$ and suppose it has Lipschitz constant $L \geq 1$. Suppose that $w \in C$ is a $(\delta, s)$-supported point in $(X, d)$, that is

$$
\inf _{p \in X}\left|\left(B\left(w, \rho_{w} / \delta\right) \backslash B\left(p, \delta \rho_{w}\right)\right) \cap C\right| \geq s .
$$


Let $w^{\prime}=f(w)$. We denote by $\rho_{w^{\prime}}$ the isolation radius of $f(w)$ with respect to $f(C)$ in $\mathbb{R}^{n}$ with the Euclidean metric. Note that $\rho_{w} / L \leq \rho_{w^{\prime}} \leq L \rho_{w}$. So

$$
f\left(B\left(w, \rho_{w} / \delta\right)\right) \subseteq B\left(w^{\prime}, L^{2} \rho_{w^{\prime}} / \delta\right)
$$

and for any $p \in X$,

$$
f\left(B\left(p, \delta \rho_{w}\right)\right) \supseteq B\left(p^{\prime}, \delta \rho_{w^{\prime}} / L^{2}\right) \supseteq B\left(q, \rho_{w^{\prime}} \delta / 2 L^{2}\right)
$$

for any $q \in X$ chosen optimally for the definition of $(\delta, s)$-supported. So $w^{\prime}$ is $\left(\delta / 2 L^{2}, s\right)$-supported in $\mathbb{R}^{n}$. As $\mathbb{R}^{n}$ carries a Benjamini-Schramm lemma with constant $c(\delta)$, it follows that $X$ carries a Benjamini-Schramm lemma with constant $c\left(\delta / 2 L^{2}\right)$.

Suppose $(X, d)$ is a metric space and $0<\epsilon<1$. Then we may consider a new distance on $X$ by taking $d^{\epsilon}(x, y):=(d(x, y))^{\epsilon}$. This new metric space $\left(X, d^{\epsilon}\right)$, often called a snowflaked version of $(X, d)$, is not in general bi-Lipschitz equivalent to the original space. Note that if $(X, d)$ has any rectifiable curves, then $\left(X, d^{\epsilon}\right)$ does not. However, this new metric is still useful due to the following theorem.

Theorem B (P. Assouad [1]). Let $(X, d)$ be a doubling metric space and $0<\epsilon<1$. Then, $\left(X, d^{\epsilon}\right)$ admits a bi-Lipschitz embedding into $\mathbb{R}^{n}$, quantitatively.

By quantitatively, we mean that the choice of $n$ and the Lipschitz constant of the embeddding both depend on $\epsilon$; however, a refinement of this theorem due to A. Naor and O. Neimann remarkably removed the dependence of $n$ on $\epsilon$ [11. Recently, G. David and M. Snipes have found a constructive and non-probabilistic proof of this independence [4].

Noting that Theorem B and Proposition 11imply that every doubling metric space can be snowflaked to become a space that carries a Benjamini-Schramm lemma, the sufficiency part of Theorem 1 will immediately follow from the proposition below.

Proposition 2. Suppose for a metric space $(X, d)$ and $0<\epsilon<1$ that $\left(X, d^{\epsilon}\right)$ carries a Benjamini-Schramm lemma. Then, $(X, d)$ carries a Benjamini-Schramm lemma.

Proof. Let $C$ be a finite set in $(X, d)$ and let $\rho_{w, \epsilon}$ be the isolation radius of $w \in C$ with respect to the metric $d^{\epsilon}$. Then,

$$
\rho_{w, \epsilon}=\rho_{w}{ }^{\epsilon} .
$$

Let $B^{\epsilon}$ stand for balls in the $d^{\epsilon}$ metric and the standard $B$ for balls in the $d$ metric. Note that

$$
\begin{aligned}
B^{\epsilon}\left(w, \rho_{w, \epsilon} / \delta\right) & =\left\{x: d^{\epsilon}(x, w)<\rho_{w, \epsilon} / \delta\right\} \\
& =\left\{x: d(x, w)<\left(\rho_{w, \epsilon}\right)^{1 / \epsilon} / \delta^{1 / \epsilon}\right\} \\
& =B\left(w, \rho_{w} / \delta^{1 / \epsilon}\right) .
\end{aligned}
$$

Also, similarly, for any $p \in X$,

$$
B^{\epsilon}\left(p, \rho_{w, \epsilon} \delta\right)=B\left(p, \rho_{w} \delta^{1 / \epsilon}\right) .
$$

Hence for $\delta>0$, the constant $c=c(X, \delta)$ needed for the Benjamini-Schramm lemma for $\left(X, d^{\epsilon}\right)$ will suffice as the appropriate constant for $\delta^{1 / \epsilon}>0$ in the BenjaminiSchramm lemma for $(X, d)$.

Now we address the necessity in Theorem 1 
Proposition 3. Suppose that the metric space $(X, d)$ is not doubling. Then the space does not carry a Benjamini-Schramm lemma.

Proof. Let $(X, d)$ be a metric space that is not doubling. Hence, for each $N \in \mathbb{N}$ there exists an $A_{N} \subset X$ where diam $A_{N}=d_{N}$ needs at least $N$ balls of radius $d_{N} / 2$ to cover $A_{N}$. Consider a cover $\mathcal{G}$ of $A_{N}$ by balls of diameter $d_{N} / 10$. By a basic covering theorem (see [7, Theorem 1.2) there is a subcover of $\mathcal{G}$, denoted $\mathcal{G}^{\prime}$, which is disjoint and $5 \mathcal{G}^{\prime}$ covers $A_{N}$. By the assumption on $A_{N}$, the number of balls in $\mathcal{G}^{\prime}$ is at least $N$. Let $x_{k}$ be the centers of the balls in $\mathcal{G}^{\prime}$ and the set $C$ the collection of these $x_{k}$ 's. We will show that each point of $C$ is $(1 / 40, N-1)$-supported.

By the disjointness of the cover $\mathcal{G}^{\prime}, d\left(x_{j}, x_{k}\right) \geq d_{N} / 10$ if $j \neq k$. Let $\delta=1 / 40$. For each $x_{k}$

$$
B\left(x_{k}, \rho_{x_{k}} / \delta\right) \supseteq B\left(x_{k},\left(d_{N} / 10\right) / \delta\right)=B\left(x_{k}, 4 d_{N}\right) .
$$

We claim that for each $k, B\left(x_{k}, \rho_{x_{k}} / \delta\right)$ contains all of the set $C$. We may assume that for $B \in \mathcal{G}^{\prime}$, the ball $5 B$ intersects the set $A_{N}$ (as $\mathcal{G}^{\prime}$ was chosen so the $5 B$ cover $\left.A_{N}\right)$. As the diam $A_{N}=d_{N}$, a ball of radius $4 d_{N}$ centered at $x_{k}$ contains all of $C$ (actually only a ball radius of $2 d_{N}$ is needed).

Now consider the quantity $\rho_{x_{k}} \delta$. As the points of $C$ are of mutual pairwise distance at least $d_{N} / 10$ apart, if $\rho_{x_{k}} \delta$ is less than $d_{N} / 20$, then for any $p \in X$ the ball $B\left(p, \rho_{x_{j}} \delta\right)$ may only contain one element of $C$. But $\rho_{x_{k}} \delta$ is less than $d_{N} / 20$ if and only if

$$
\rho_{x_{k}}<2 d_{N}
$$

This is true because, as already noted, the ball $B\left(x_{k}, 2 d_{N}\right)$ contains all of the set C.

Hence each point of the set $C$ is $(1 / 40, N-1)$-supported. No metric space carrying a Benjamini-Schramm lemma may contain such sets for each $N \in \mathbb{N}$.

Taken together, Propositions 11, 2, and 3 prove Theorem 1.

\section{ACKNOWLEDGEMENTS}

The author would like to thank Steffen Rohde for encouraging work in this direction, the enthusiastic audience of the Saint Louis University analysis seminar for their questions and comments, especially Bryan Clair, and the anonymous referee for a close reading and some helpful suggestions which improved the exposition.

\section{REFERENCES}

[1] Patrice Assouad, Plongements lipschitziens dans $\mathbf{R}^{n}$ (French, with English summary), Bull. Soc. Math. France 111 (1983), no. 4, 429-448. MR763553 (86f:54050)

[2] Itai Benjamini and Nicolas Curien, On limits of graphs sphere packed in Euclidean space and applications, European J. Combin. 32 (2011), no. 7, 975-984, DOI 10.1016/j.ejc.2011.03.016. MR2825530(2012g:05050)

[3] Itai Benjamini and Oded Schramm, Recurrence of distributional limits of finite planar graphs, Electron. J. Probab. 6 (2001), no. 23, 13 pp. (electronic), DOI 10.1214/EJP.v6-96. MR.1873300 (2002m:82025)

[4] G. David and M. Snipes, A non-probabilistic proof of the Assouad embedding theorem with bounds on the dimension, Anal. Geom. Metr. Spaces 1 (2013), 36-41. MR3108866

[5] Ori Gurel-Gurevich and Asaf Nachmias, Recurrence of planar graph limits, Ann. of Math. (2) 177 (2013), no. 2, 761-781, DOI 10.4007/annals.2013.177.2.10. MR3010812

[6] J. T. Gill and S. Rohde, On the Riemann surface type of random planar maps, Rev. Mat. Iberoam. 29 (2013), no. 3, 1071-1090, DOI 10.4171/RMI/749. MR.3090146 
[7] Juha Heinonen, Lectures on analysis on metric spaces, Universitext, Springer-Verlag, New York, 2001. MR:1800917 (2002c:30028)

[8] Jouni Luukkainen, Assouad dimension: antifractal metrization, porous sets, and homogeneous measures, J. Korean Math. Soc. 35 (1998), no. 1, 23-76. MR.1608518 (99m:54023)

[9] John M. Mackay and Jeremy T. Tyson, Conformal dimension, Theory and application. University Lecture Series, vol. 54, American Mathematical Society, Providence, RI, 2010. MR2662522(2011d:30128)

[10] H. Namazi, P. Pankka and J. Souto, Distributional limits of Riemannian manifolds and graphs with sublinear genus growth, Geom. Funct. Anal. 24 (2014), no. 1, 322-359, DOI 10.1007/s00039-014-0259-6. MR.3177385

[11] Assaf Naor and Ofer Neiman, Assouad's theorem with dimension independent of the snowflaking, Rev. Mat. Iberoam. 28 (2012), no. 4, 1123-1142, DOI 10.4171/RMI/706. MR2990137

Department of Mathematics and Computer Science, Saint Louis University, 220 N. Grand Boulevard, St. Louis, Missouri 63103

E-mail address: jgill5@slu.edu 